\title{
Evolution of Lowest Supply Voltage and Hysteresis Phenomena in Irradiated Analog CMOS Switches
}

\author{
F. J. Franco, Y. Zong and J. A. Agapito \\ Departamento Física Aplicada III, \\ Facultad de CC. Físicas, Universidad Complutense de Madrid, \\ Madrid (SPAIN) \\ monti@fis.ucm.es, agapito@fis.ucm.es, yzong@fis.ucm.es
}

\author{
J. Casas-Cubillos, M.A. Rodríguez-Ruiz \\ CERN, AT Division, \\ Geneva (SWITZERLAND) \\ juan.casas-cubillos@cern.ch \\ miguel.angel.rodriguez.ruiz@cern.ch
}

\begin{abstract}
Radiation tests on CMOS analog switches were done in order to select the most tolerant device for the future use in the cryogenic system of LHC. After the irradiation, the devices showed some interesting changes related to the power supplies: An increase in the lowest supply voltage capable of biasing correctly the devices, some devices cannot work with TTL logic levels and hysteresis phenomena appear. ${ }^{1}$
\end{abstract}

Index Terms-Analog switches, COTS, TID.

\section{INTRODUCTION}

An analog switch is a non-linear resistor controlled by an external logic input. For an ideal switch, the impedance is infinite or 0 depending on the value of the logic input " 0 " or "1" [1].

The analog stage of a switch consists of a couple of NMOS and PMOS transistors with source and the drain terminal linked together. Their gates are connected to two complementary outputs of the logic stage that simultaneously carry them into cut-off or lineal state. The use of two transistors is intended to minimize the non-linearity of the resistance value of the switches. The logic stage of an analog switch consists of several parts: A driver, to transform the primary logic input into hard logic values; a level shifter, to change the logic values into voltages more appropriated to bias the analog channel and, finally, a CMOS inverter to obtain the complementary value of the main output. Fig. 1 and 2 shows the typical structure of an analog switch.

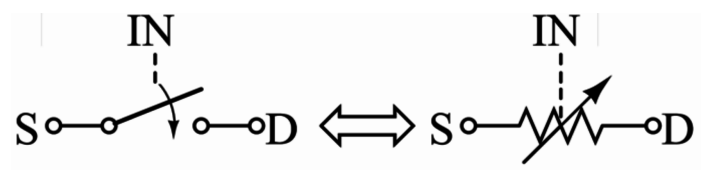

Figure 1. Electric symbol of an analog switch (a) and the equivalent nonlinear resistor $(b)$.

Radiation tests on analog switches were carried out in order to propose their use in the development of the electronic instrumentation of the cryogenic system of the future Large Hadron Collider (LHC), at the European Laboratory for Nuclear Research (CERN). In this harsh environment,

\footnotetext{
${ }^{1}$ This work was supported by the cooperation agreement K476/LHC between CERN and UCM, by the Spanish Research Agency CICYT (FPA200200912) and partially supported by ITN.
}

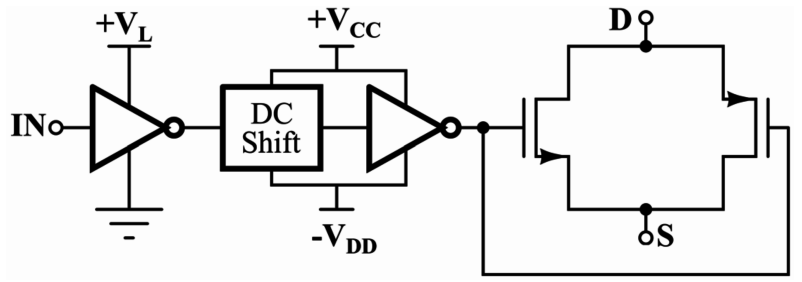

Figure 2. Typical structure of an analog switch. The first inverter converts the input IN into hard logic levels, 0 or $+\mathrm{V}_{L}$. Then, a new stage transforms these values into $+V_{C C}$ and $-V_{D D}$, more appropriate to bias the gate of the MOS transistors making the analog channel of the switch.

electronic devices are expected to receive a neutron fluence of $5 \cdot 10^{13}$ nůcm $^{-2}$ and a total gamma dose of several hundreds of Gy(air). Therefore, radiation tests were adapted to this kind of environment.

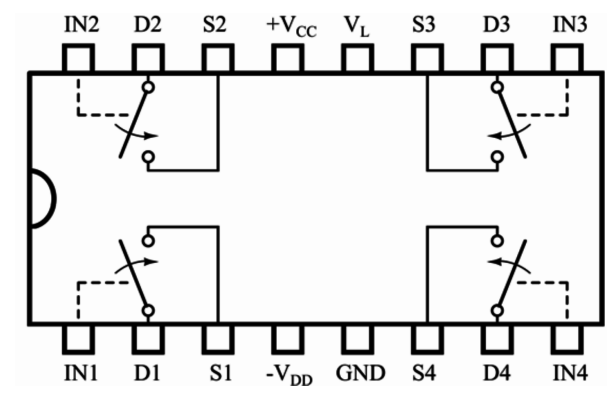

Figure 3. Usual 4xNO SPST analog switches.

The tested devices were ADG212 and ADG412 from Ana$\log$ Devices, DG212 and MAX332 from Maxim, and DG212 and DG412, from Vishay. These devices were 4xNO-Single Pole /Single Track, which means that there are 4 switches in the chip, Normally Open if $I N=0$, and with one only control input and an only channel (Fig. 3). They were irradiated in the neutron facility of the Portuguese Research Reactor [2], where the previous value of neutron fluence is reached in the center of the cavity after five sessions of $12 \mathrm{~h}$ every day. During the nights, the reactor shut down for technical reasons. Also, the vestigial gamma dose is about 1.2-2 kGy (air). Due to these values are several times the expected value of gamma radiation, the tests are valid as NIEL tests and as accelerated 
Table I

TOTAL RADIATION DOSE OF THE DEVICES

\begin{tabular}{lccc} 
& NIEL & TID & D.R. \\
\hline ADG212 & 6.39 & 1720 & 28.7 \\
\hline ADG412 & 6.39 & 1720 & 28.7 \\
\hline DG212 (Maxim) & 4.91 & 1580 & 26.3 \\
\hline MAX332 & 4.91 & 1580 & 26.3 \\
\hline DG212 (Vishay) & 5.61 & 1660 & 27.7 \\
\hline DG412 (Vishay) & 5.61 & 1660 & 27.7 \\
\hline & $\cdot 10^{13} \mathrm{n} \cdot \mathrm{cm}^{-2}$ & Gy(air) & Gy(air)/h \\
\hline
\end{tabular}

TID tests, as CERN irradiation protocol suggests [3].

The displacement damage associated to the neutron beam causes an increase of the serial resistance of the analog switch [4]. In addition, the ionizing radiation creates positive charges in the gate oxide and in the epitaxial $\mathrm{SiO}_{2}$ [5]. The consequence is the shift of MOSFET threshold voltages, the appearance of leakage currents and the increase of power consumption. The main goal of this paper is to find out how the ionizing radiation affects to the working values of the power supplies. Other points related to these tests have been studied in other works [6], [7].

During the irradiation, the devices were biased with $\pm 15 \mathrm{~V}$ to track the evolution of the resistance of the analog channel and the appearance of leakage currents. Later, the devices were isolated for safety for about a month at room temperature until the disappearance of the active isotopes. The total radiation dose received by the samples is shown in Table I.

\section{INFLUENCE OF TID ON THE LOWEST SUPPLY VOLTAGES}

All the devices consist of four normally open switches and each device needs three power supplies: $+\mathrm{V}_{C C},-\mathrm{V}_{D D}, \mathrm{~V}_{L}$. Digital and analog ground coincide at the same node, GND. The switch can be biased either by a unipolar power supply $\left(-\mathrm{V}_{D D}=0 \mathrm{~V}\right)$ or by a bipolar one $\left(-\mathrm{V}_{D D}=-\mathrm{V}_{C C}\right)$. The logic voltage $\mathrm{V}_{L}$ defines the HIGH logic value. There are two possibilities to select the value of this parameter. First of all, $\mathrm{V}_{L}=5 \mathrm{~V}$ and the switch is TTL compatible. The second option is to define the HIGH logic value as $\mathrm{V}_{L}=+\mathrm{V}_{C C}$ and this case denominated in this paper "CMOS compatible". Obviously, if $+\mathrm{V}_{C C}=5 \mathrm{~V}$, there is no difference between both cases. In short, once the value of $+\mathrm{V}_{C C}$ is selected, there are four possibilities to bias the switches: Unipolar TTL, Unipolar CMOS, Bipolar TTL and Bipolar CMOS. The exception is when $+\mathrm{V}_{C C}=5 \mathrm{~V}$ since only the TTL logic supplies are defined.

Tables IIa-b show the switching threshold voltage of the irradiated switches depending on the bias and on the logic compatibility. The first column in the left side shows the values of the power supplies and the logic compatibility. There are two columns for each switch. The column on the left shows the values before the irradiation and the other one, on the right, the values after it. E.g., the DG212 sample from Maxim switched on at $1.72 \mathrm{~V}$ before the irradiation with a unipolar
$15 \mathrm{~V}$ power supply but, after it, this value changed up to 3.91 $\mathrm{V}$.

Other values of the table after irradiation should be explained. First of all, the device could not switch on and off at certain voltage supplies. Therefore, the switch must be always open or closed (e.g. ADG212 from Analog Devices with any power supply). In other cases, there are a pair of values that represents two different behaviors: If they are underlined, there is a hysteresis phenomenon (e. g., the irradiated MAX332 sample with $\pm 15 \mathrm{~V}$ power supply). In this device, the pair of values were 3.91 and $4.46 \mathrm{~V}$. The devices switch on when the logic input is increasing and reaches $4.46 \mathrm{~V}$ and switch off when it decreases below $3.91 \mathrm{~V}$. The other possibility is that the values are not underlined (E. g., the MAX332 sample with a unipolar $15 \mathrm{~V}$ power supply before the irradiation). This fact means that the action of switching from on to off is not sharp but very soft from the first value up to the second one. Hysteresis does not exist.

Tables IIa-b show the minimum power supply needed to have a "good" open state. A good open state is that in which the switch resistance is extremely high. In this work, we have chosen a value of $100 \mathrm{M} \Omega$ as the lowest value that a resistance must have to be considered open circuit. Some devices had lost the possibility of changing from open to closed state, so this fact has been pointed out in the table as "closed".

Finally, tables IIa-b provide information about the minimum power supply that the devices need to switch on and off. In other words, the open and off states are well defined if the device is biased with that value of power supply. In this paper, we have postulated that a switch correctly works if $R_{O P E N}>100 \cdot R_{C L O S E D}$. According to this criterion, a device can switch even if $R_{O P E N}$ is not large enough to be considered a good open circuit.

\section{ANALYSIS OF DATA}

The preceding set of data allows us to find out some interesting characteristics of the irradiated switches. They are summarized in the following sections.

\section{A. Increase of lowest supply voltage}

According to the tables II-II, a not irradiated switch can work even with unipolar power supplies in the order of 4$5 \mathrm{~V}$. However, this feature is not present in the irradiated switches. For instance, Table IIa shows the DG212 sample from Maxim does not work unless the value of is at least $15 \mathrm{~V}$. Similar results can be obtained from the other samples except ADG212, which is continuously closed. Also, according to Table IIa-b, the value of power supply needed to have a good difference between the open and closed states are much higher. On the contrary, the power supply that defines a good open state has hardly change in all the samples except DG412 from Vishay.

The values of ADG412 shown in Table IIa are interesting enough since we can suspect the existence of a pair of minimum power supplies instead of only one. The facts that support this statement are the following: First of all, the switch 
Table II

SWITCHING THRESHOLD VOLTAGE OF IRRADIATED SWITCHES

\begin{tabular}{|c|c|c|c|c|c|c|}
\hline & \multicolumn{2}{|c|}{ ADG212 (Analog) } & \multicolumn{2}{|c|}{ ADG412 (Analog) } & \multicolumn{2}{|c|}{ DG212 (Maxim) } \\
\hline Supply & Not Irr. & Irrad. & Not Irr. & Irrad. & Not Irr. & Irrad. \\
\hline $0-5 \mathrm{~V}$ & 1.64 & Closed & $1.6-2.4$ & Open & 1.34 & Open \\
\hline $\pm 5 \mathrm{~V}$ & 1.61 & Closed & $1.6-2.4$ & Closed & 1.34 & Open \\
\hline $0-10 \mathrm{~V}$ TTL & 1.65 & Closed & 1.75 & Closed & 1.52 & Open \\
\hline $0-15 \mathrm{~V}$ TTL & 1.57 & Closed & 1.69 & Open & 1.72 & 3.91 \\
\hline $\pm 10 \mathrm{~V} \mathrm{TTL}$ & 1.65 & Closed & 1.75 & Closed & 1.52 & $\underline{2.81-4.70}$ \\
\hline $\pm 15 \mathrm{~V} \mathrm{TTL}$ & 1.57 & Closed & 1.67 & Open & 1.72 & $3.76-3.82$ \\
\hline $0-10 \mathrm{~V}$ CMOS & 1.65 & Closed & 3.03 & Closed & 1.52 & Open \\
\hline $0-15 \mathrm{~V}$ CMOS & 1.57 & Closed & $4.02-4.04$ & $1.77-1.81$ & 1.72 & 3.91 \\
\hline $\pm 10 \mathrm{~V} \mathrm{CMOS}$ & 1.65 & Closed & 3.02 & Closed & 1.52 & $2.81-4.70$ \\
\hline \multirow[t]{2}{*}{ $\pm 15 \mathrm{~V} \mathrm{CMOS}$} & 1.57 & Closed & $\underline{3.94-4.02}$ & $1.62-1.87$ & 1.72 & $3.76-3.82$ \\
\hline & \multicolumn{2}{|c|}{ MAX332 (Maxim) } & \multicolumn{2}{|c|}{ DG212 (Vishay) } & \multicolumn{2}{|c|}{ DG412 (Vishay) } \\
\hline Supply & Not Irr. & Irrad. & Not Irr. & Irrad. & Not Irr. & Irrad. \\
\hline $0-5 \mathrm{~V}$ & $1.37-1.52$ & Open & 1.54 & Open & 2.17 & Closed \\
\hline $\pm 5 \mathrm{~V}$ & $1.38-1.52$ & Open & 1.54 & Open & $2.14-2.16$ & Closed \\
\hline $0-10 \mathrm{~V}$ TTL & 1.56 & Open & $1.54-1.63$ & $1.47-1.51$ & 1.58 & Closed \\
\hline $0-15 \mathrm{~V}$ TTL & $1.56-2.19$ & $3.97-4.69$ & $1.57-1.89$ & $1.56-1.67$ & $1.26-1.28$ & Closed \\
\hline $\pm 10 \mathrm{~V} \mathrm{TTL}$ & 1.67 & $2.75-4.62$ & $1.54-1.61$ & 1.36 & $1.58-1.60$ & Closed \\
\hline $\pm 15 \mathrm{~V} \mathrm{TTL}$ & $1.77-2.37$ & 4.03-4.04 & $\underline{1.56-1.68}$ & $\underline{1.49-1.58}$ & 1.26-1.28 & 11 \\
\hline 0-10V CMOS & 1.67 & Open & $3.02-3.09$ & 2.88 & 4.39 & Closed \\
\hline $0-15 \mathrm{~V}$ CMOS & $1.82-2.37$ & $\underline{3.97-4.69}$ & 4.54-4.65 & $\underline{4.51-4.56}$ & 6.80-6.85 & Closed \\
\hline $\pm 10 \mathrm{~V}$ CMOS & 1.67 & $2.79-4.62$ & $3.03-3.07$ & $2.81-283$ & $4.31-4.38$ & Closed \\
\hline $\pm 15 \mathrm{~V}$ CMOS & $1.83-2.45$ & $3.91-4.46$ & $4.53-4.60$ & $4.41-4.47$ & 6.64-6.85 & 11 \\
\hline
\end{tabular}

is open with a unipolar $+5 \mathrm{~V}$ power supply but, if this voltage increases up to $10 \mathrm{~V}$ or the supply becomes bipolar, the switch is always closed. Also, if $+\mathrm{V}_{C C}$ rises, the device can switch on and off.

This suggests that the analog channel can be correctly biased with a voltage lower than that one needed to bias the logic stage. There is a lowest supply voltage for the analog channel and another one for the logic stage. Thus, the analog channel is open with $0-5 \mathrm{~V}$ since the supply voltage is not high enough to bias it. A higher supply voltage can correctly bias the channel but cannot do the same with the logic stage and, only when the voltage supply increases up to $+15 \mathrm{~V}$, the logic stage work and the switch works.

Overall, the higher the difference between the power supplies, the more likely the device work. Only the ADG412 sample infringes this general behavior: It works with an unipolar $+15 \mathrm{~V}$ power supply but does not with $\pm 10 \mathrm{~V}$.

\section{B. Disability of TTL compatible logic. Shift of switching threshold voltage}

Most digital devices can identify the HIGH logic level with $+5 \mathrm{~V}$ and the LOW level with $0 \mathrm{~V}$. The not irradiated samples, except DG412 from Vishay, could switch on and off with this voltage levels. However, the irradiated ADG412 sample is a device that can switch with $1.6 \mathrm{~V}$ if $\mathrm{V}_{L}=+\mathrm{V}_{C C}$ but loses this feature if $\mathrm{V}_{L}=5 \mathrm{~V}$. Therefore, although the switch is activated by TTL compatible levels, its logic power supply must be much higher than $5 \mathrm{~V}$.

In addition to this fact, the switching threshold voltages shift during the irradiation. Sometimes it rises, as DG212 and MAX332 from Maxim, but it decreases in other switches as ADG212 and ADG412. In any case, their values depend on the value of the logic power supply.

\section{Hysteresis}

One interesting fact that takes place in the irradiated sample is the appearance of hysteresis. This phenomenon can appear in not irradiated devices but the cycle is usually very narrow (A tenth of volt). However, it appears and becomes important in the irradiated switches. The most representative samples of this behavior are the switches from Maxim. In these devices, the width of the hysteresis cycle is about $2 \mathrm{~V}$.

Three devices showed hysteresis after the irradiation: DG212 and MAX332 from Maxim and DG212 from Vishay. In the samples of Maxim, this phenomenon is observed only when the difference between the power supplies is high enough $( \pm 10$ and $\pm 15 \mathrm{~V}$ ). On the contrary, the hysteresis in the sample from Vishay is related to the value of the positive power supply $(0-15 \mathrm{~V}$ and $\pm 15 \mathrm{~V})$.

In short, the hysteresis phenomenon might appear in irradiated devices biased with high power supplies. Moreover, there is not any dependence on the value of the logic power supply. 
Table III

VALUES OF THE LOWEST POWER SUPPLIES TO HAVE A “GOOD” OPEN STATE (V). THE FIRST COLUMN IN THE LEFT SHOWS THE KIND OF POWER SUPPLIES (UNIPOLAR OR BIPOLAR) AND THE HIGH LOGIC LEVEL $\left(\mathrm{TTL}=5 \mathrm{~V}, \mathrm{CMOS}=+\mathrm{V}_{C C}\right.$ ).

\begin{tabular}{ccccccc} 
& \multicolumn{1}{c}{ ADG212 (Analog) } & \multicolumn{2}{c}{ ADG412 (Analog) } & \multicolumn{2}{c}{ DG212 (Maxim) } \\
\hline Power Supply & Not Irr. & Irrad. & Not Irr. & Irrad. & Not Irr. & Irrad. \\
\hline Unipolar TTL & 4.66 & Closed & 4.79 & Closed & 4.75 & 4.79 \\
\hline Unipolar CMOS & 4.64 & Closed & 4.75 & Closed & 4.73 & 4.79 \\
\hline Bipolar TTL & \pm 4.68 & Closed & \pm 4.78 & Closed & \pm 4.73 & \pm 4.72 \\
\hline Bipolar CMOS & \pm 4.71 & Closed & \pm 4.72 & Closed & \pm 4.76 & \pm 4.72 \\
\hline & MAX332 (Maxim) & \multicolumn{2}{c}{ DG212 (Vishay) } & \multicolumn{2}{c}{ DG412 (Vishay) } \\
\hline Power Supply & Not Irr. & Irrad. & Not Irr. & Irrad. & Not Irr. & Irrad. \\
\hline Unipolar TTL & 4.91 & 5.15 & 5.23 & 4.82 & 4.92 & Closed \\
\hline Unipolar CMOS & 5.22 & 4.72 & 5.25 & 4.79 & 4.79 & Closed \\
\hline Bipolar TTL & \pm 5.06 & \pm 5.16 & \pm 5.28 & \pm 4.78 & \pm 4.83 & Closed \\
\hline Bipolar CMOS & \pm 5.17 & \pm 5.31 & \pm 5.18 & \pm 4.78 & \pm 4.83 & Closed \\
\hline
\end{tabular}

Table IV

LOWEST POWER SUPPLY TO HAVE OPEN AND CLOSED STATES (V).

ADG212 (Analog)

ADG412 (Analog)

DG212 (Maxim)

\begin{tabular}{ccccccc}
\hline Power Supply & Not Irr. & Irrad. & Not Irr. & Irrad. & Not Irr. & Irrad. \\
\hline Unipolar TTL & 3.1 & Closed & 2.2 & Closed & 3.2 & 15.1 \\
\hline Unipolar CMOS & 3.1 & Closed & 3.1 & Closed & 3.2 & 15.1 \\
\hline Bipolar TTL & \pm 2.2 & Closed & \pm 3.1 & Closed & \pm 2.7 & \pm 8.4 \\
\hline Bipolar CMOS & \pm 2.1 & Closed & \pm 4.7 & Closed & \pm 3.0 & \pm 8.4 \\
\hline \multicolumn{7}{r}{} \\
MAX332 (Maxim) & \multicolumn{2}{c}{ DG212 (Vishay) } & \multicolumn{2}{c}{ DG412 (Vishay) } \\
\hline Power Supply & Not Irr. & Irrad. & Not Irr. & Irrad. & Not Irr. & Irrad. \\
\hline Unipolar TTL & 3.4 & 14.5 & 3.4 & 9.6 & 2.2 & Closed \\
\hline Unipolar CMOS & 3.4 & 14.5 & 2.5 & 9.7 & 3.1 & Closed \\
\hline Bipolar TTL & \pm 3.2 & \pm 7.7 & \pm 3.2 & $\pm 5.2-7.3$ & \pm 3.1 & Closed \\
\hline Bipolar CMOS & \pm 3.2 & \pm 7.7 & \pm 2.4 & $\pm 5.2-8.3$ & \pm 4.7 & Closed \\
\hline
\end{tabular}

\section{Similarity among switches}

The results shown in previous tables are related to the first switch of each device. However, similar evolutions were found in the other switches of the sample. Hence, if there is a shift in the switching voltage or the appearance of a hysteresis cycle in the first device, the same phenomenon is found in the other three switches. The only difference is that there is a little scattering in the voltage values although the difference among the values is not higher than $0.1 \mathrm{~V}$.

\section{DISCUSSION}

The increase of the lowest supply voltage is doubtlessly related to the shift of the threshold voltage of the MOS transistors. This phenomenon happens in the analog channel and in the logic stage. The exact explanation is difficult to find out since the whole internal structure of the device is not known but this behavior can be easily understood with several examples. For instance, let us assume that the source $S$ of the analog channel of fig. 2 is grounded. Also, the value of $\mathrm{V}_{D}$ is usually very close to $\mathrm{V}_{S}$. So, we can accept that
$V_{D} \approx V_{S}=0$. In this situation, the transistors of the analog channel are in the linear zone if:

$$
V_{C C}>V_{T H, N} \& V_{E E}>\left|V_{T H, P}\right|
$$

If the irradiation is not too fast, the values of $V_{T H, N}$ and $\left|V_{T H, P}\right|$ will increase and the growth of lowest supply voltage is easily explained from eq. 1 .

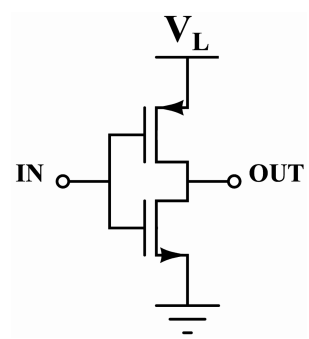

Figure 4. Simplest CMOS inverter.

Also, the use of simple examples is a way to understand the evolution of switching threshold voltage and the disability of 


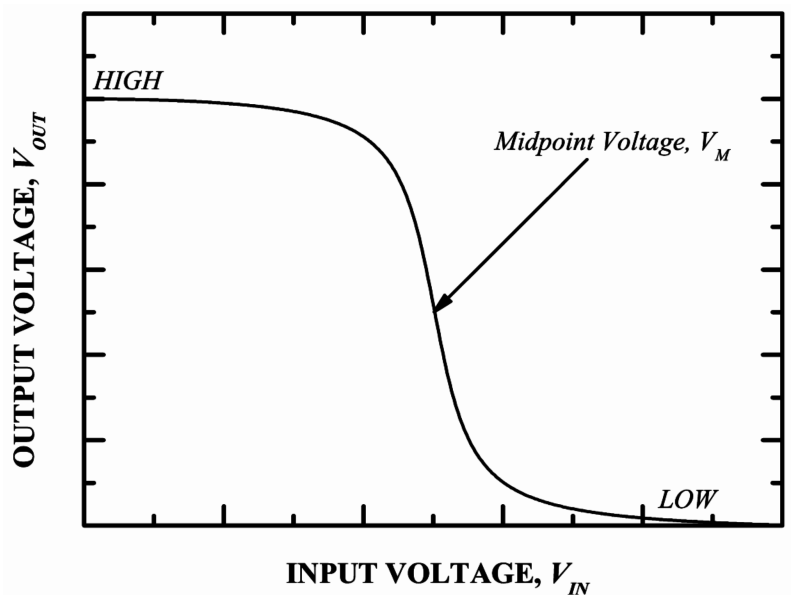

Figure 5. Voltage transfer characteristics of inverter of fig. 4.

TTL logic levels. In this case, we will use the classical CMOS inverter, as that shown in fig. 4 , to explain these phenomena. The transfer function between the input and output voltage, $\mathrm{V}_{I N}$ and $\mathrm{V}_{O U T}$, is similar to that sketched in fig. 5. If we assume that the switching voltage of this device is the midpoint voltage, $\mathrm{V}_{M}$, its value will be, according to [8]:

$$
V_{M}=\frac{V_{L}+V_{T H, P}+V_{T H, N} \cdot \sqrt{\frac{k_{N}}{k_{P}}}}{1+\sqrt{\frac{k_{N}}{k_{P}}}}
$$

$k_{N}$ and $k_{P}$ are the transconductance of the transistors. In some cases, like the DG412 switch from Vishay Siliconix, the first inverter of fig. 2 is similar to the inverter of fig. 4 and eq. 2 depicts perfectly its behavior. We do not know the internal structure of the other devices but it is likely that the inverter should be similar to fig. 4. During the irradiation, $\mathrm{V}_{T P}$ and $\mathrm{V}_{T N}$ shift so the value of $\mathrm{V}_{M}$ must be different from the initial one. The shift of the switching threshold voltage has been found as well by other authors [9].

However, there is still a mathematical condition to deal with. Obviously, the value of $\mathrm{V}_{M}$ must be placed between 0 and $\mathrm{V}_{L}$ since it is the midpoint value. Thus, from this condition the following couple of inequations are deduced:

$$
\begin{gathered}
\sqrt{\frac{k_{N}}{k_{P}}} \cdot V_{L}>V_{T H, P}+\sqrt{\frac{k_{N}}{k_{P}}} \cdot V_{T H, N} \\
V_{L}>-V_{T H, P}-\sqrt{\frac{k_{N}}{k_{P}}} \cdot V_{T H, N}
\end{gathered}
$$

Before the irradiation, the values of $\mathrm{V}_{T H, N}$ and $\mathrm{V}_{T H, P}$ are not very large and the preceding conditions are true. However, during the irradiation, eq. 3 or 4 might become false due to the shift of both parameters. Also, the lower the value of $\mathrm{V}_{L}$, the easier the fact that some of the rules is broken. Thus, the impossibility of the use of TTL supply voltages is explained.

The appearance of hysteresis voltage cannot be explained but it is not a strange fact in digital circuits. Some digital networks show hysteresis as, for example, the Schmitt CMOS inverter, where the hysteresis minimizes the action of the noise of the logic input [8]. Therefore, the shift of the transistor parameters and the appearance of parasitic NMOS transistors can modify the characteristics and the topology of the logic stage. Thus, the appearance of hysteresis phenomenon is not as strange as it may seem.

\section{CONCLUSIONS}

The action of TID on the power sources that supplies analog CMOS switches is not only restricted to the growth of supply currents but also leads to the increase of lowest supply voltage. Thus, the minimum values that the manufacturer provides in the devices' data sheet are no longer true. Moreover, the use of low voltage supplies ( $3.3 \mathrm{~V}$ or less) is not advised in this kind of devices.

In addition, the switching threshold voltage shifts because of total ionizing dose and, thus, the appearance of hysteresis phenomena becomes possible.

These results were obtained from analog CMOS switches. However, a similar behavior might happen in other kinds of CMOS devices.

\section{REFERENCES}

[1] P. Horowitz, The Art of Electronics, 2nd ed. Cambridge University Press, 1990.

[2] A. C. Fernandes, I. C. Goncalves, J. G. Marques, J. Santos, A. J. G. Ramalho, and M. Osvay, "Mixed-field dosimetry of a fast neutron beam at the Portuguese Research Reactor for the irradiation of electronic circuits - measurements and calculations," in Proc. 11th Intl. Symp. on Reactor Dosimetry, Aug. 2002, pp. 19-23.

[3] “ATLAS Standard Radiation Test Methods, Appendix 2," [online] Available: http://atlas.web.cern.ch/Atlas/GROUPS/FRONTEND/radhard.htm.

[4] G. C. Messenger, "A summary review of displacement damage from high energy radiation in silicon semiconductors and semiconductor devices," IEEE Transactions on Nuclear Science, vol. 39, no. 3, pp. 468-473, Jun 1992.

[5] J. R. Srour and J. M. McGarrity, "Radiation Effects on Microelectronics in Space," Proceedings of the IEEE, vol. 76, no. 11, pp. 1443-1469, November 1988.

[6] J. A. Agapito et al., "Radiation test on commercial instrumentation amplifiers, analog switches and DACs," in Proc. 7th Workshop for LHC Experiments, 2001, pp. 113-118.

[7] Y. Zong, F. J. Franco, and J. A. Agapito, "Using Optimization Techniques to Characterize Irradiated CMOS Switches," in to be presented at the RADECS2004 Workshop, Sept. 22-24 2004.

[8] T. A. DeMassa and Z. Ciccone, Digital Integrated Circuits. John Wiley and Sons, 1996, ch. 26.

[9] D. Larsen, P. Welling, and W. Tsacoyeanes, "The Effects of Ionizing Radiation on the Honeywell HTMOS High-Temperature Linear CMOS Technology," in IEEE Radiation Effects Data Workshop, Jul. 1996, pp. $55-61$. 